\title{
Growing Degree Day Calculation Method Comparison between Two Methods in the Northern Edge of the US Corn Belt
}

\author{
F. Adnan Akyüz ${ }^{1}$ and Joel K. Ransom \\ North Dakota State University, Fargo, ND
}

\begin{abstract}
The use of environmental temperature and its effects on plant development have been useful in determining growth stages of plants. This paper compares two Corn Growing Degree Day (GDD) calculation methods that are widely used in the US and why one is more suitable in the northern edge of the US corn-belt areas than the other. The comparison between the two accumulated GDD calculations for corn during the last 67-year period from 1948 to 2014 growing seasons for Fargo, ND, indicates that one method systematically underestimates accumulated GDDs during the days when maximum temperatures are above and minimum temperatures are below the base temperature of $50^{\circ} \mathrm{F}$. Furthermore, the ratio of the difference between the two seasonal accumulations to the required accumulated GDD necessary to mature the type of corn grown in this area becomes more significant than those grown in other parts of the US where corn requires higher seasonal GDD accumulations.
\end{abstract}

\section{Introduction}

Growing Degree Day is the accumulated heat unit above a certain threshold at which plants will start developing. It is the best known method to estimate the plant development stage (Miller et al. 2001). Each plant has its own base, or low threshold temperature. For corn, this threshold temperature is $50^{\circ} \mathrm{F}$ or $10^{\circ} \mathrm{C}$. Corn also has an upper threshold value of $86^{\circ} \mathrm{F}$ or $30^{\circ} \mathrm{C}$ (Gilmore and Rogers, 1958; Badh and Akyuz, 2010). It is assumed that the development rate between these two threshold values is constant. In other words, there is a linear relationship between the heat units accumulated within these thresholds and the plant's development rate. Furthermore, most GDD algorithms are designed to ignore the accumulation of the heat units when the temperature is out of the developmental range set by the upper and the lower threshold limits. The GDD method usually predicts the actual growth stages better than the "days" method which relies on number of days passed after a certain developmental stage, such as emergence and maturity (Miller et al. 2001).

This paper addresses the difference between two methods. The first method will be referred to as "Method 1", and the second method will be referred to as "Method 2" in this paper. Both methods will be discussed in detailed in the next section.

The second method differs slightly from the first and is adopted by the North Dakota Agricultural Weather Network (NDAWN) Center. The NDAWN Center is comprised of 80 automated weather stations observing weather variables in North Dakota, western Minnesota along the Red River Valley and eastern Montana to assist agricultural communities with day-to-day decision tools related to herbicide, pesticide and insecticide timing based on GDD methodology for different crops (NDAWN, 2015). The details of the comparison and results are discussed in the following sections.

\section{Methodology and Algorithm}

The Growing Degree Day accumulation can be calculated using the following equation.

$G D D=\int_{t_{1}}^{t_{2}} \theta(t) d t$

Eq. 1

${ }^{1}$ Corresponding Author Address: North Dakota State University. Department 7521, PO Box: 6050, Fargo, ND 58108-6050. Email: Adnan.Akyuz@ndsu.edu 
Where GDD is the accumulated growing degree days between $t_{1}$ and $t_{2}, d t$ is usually a 24-hour period. $\theta$ is a heat accumulation factor that accounts for plant development which is a function of time, $t$. It is usually assumed that the development rate is zero below the lower threshold temperature, $T_{l}$, and above the upper threshold temperature, $T_{u}$, where the development rate is maximum. The equation above can be simplified as a summation of the heat accumulation in small time increments. The heat accumulation in the graphic below (Figure 1 ) is proportional with the shaded area, $A$, under the temperature curve above the base or the lower threshold temperature, $T_{l}$, and below the upper threshold temperature, $T_{u}$. In Figure 1, vertical axis is temperature and the horizontal axis is time. $T_{x}$ and $T_{n}$ are daily maximum and minimum temperatures respectively. On the horizontal axis, $t_{1}$ and $t_{2}$ represent the beginning and end of the accumulation period ( 24 hours in this case). In theory, the finite time increment, $d t$, should be the shortest time period possible to better represent the area, $A$. However, the magnitude of the time increment is limited to the observation frequency.

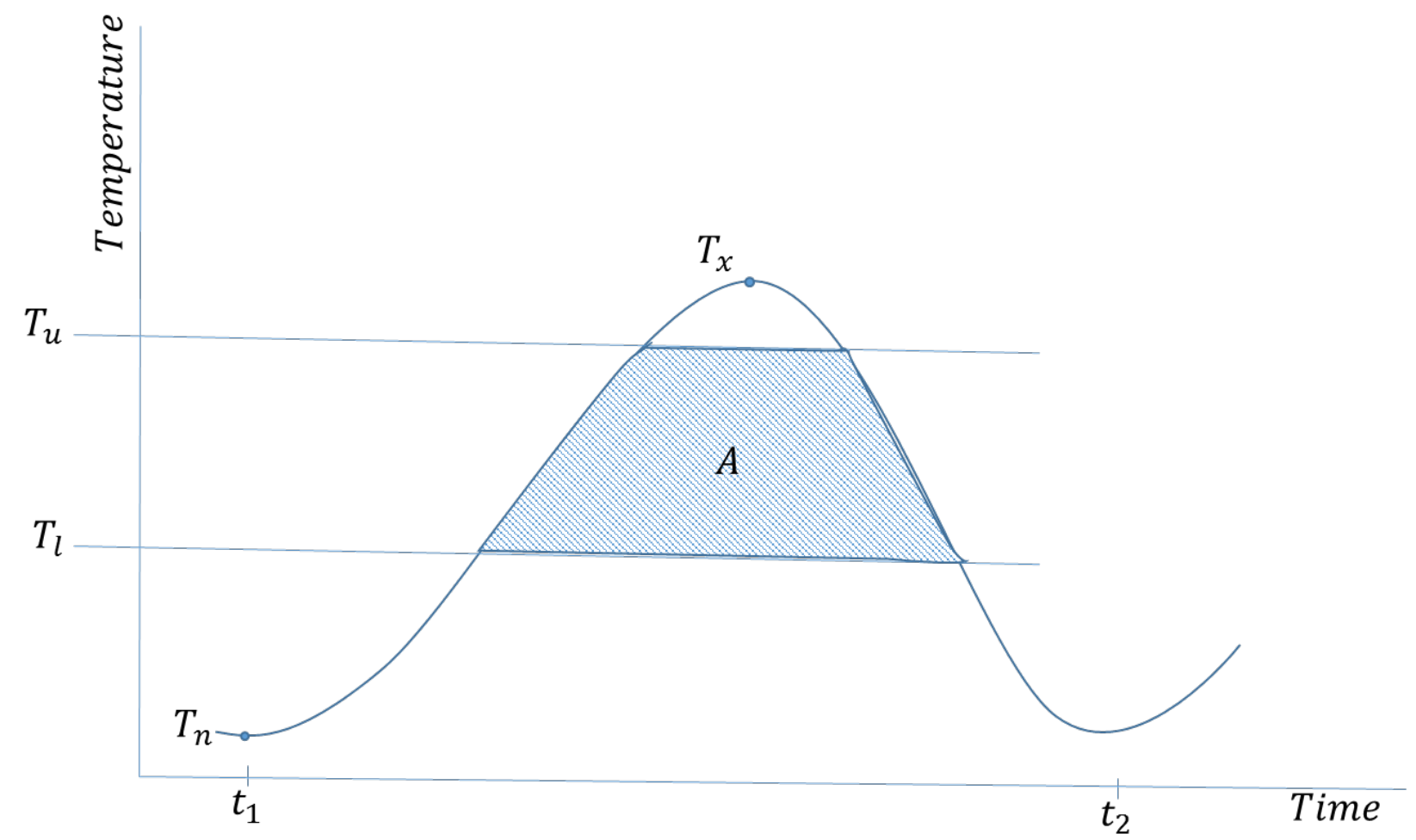

Figure 1. A Typical Diurnal Temperature Trace in Relation to Heat Accumulation for Degree Day Calculations.

Unfortunately, most historical climate data for wide portions of the country in the US only contain maximum and minimum temperature data during a 24-hour period. Therefore, the GDD calculations for these locations became limited to utilizing maximum and minimum temperatures only during a 24-hr period. For this reason the integral in Eq. 1 can be simplified by the following approximation

$G D D=\frac{T_{x}+T_{n}}{2}-T_{l}$

Eq. 2

The first term in Eq. $2\left(\frac{T_{x}-T_{n}}{2}\right)$ is the daily average temperature calculated from the daily maximum and minimum temperatures. The daily accumulated GDD is calculated by simply subtracting the lower 
threshold temperature from the daily average temperature. For seasonal accumulation, the daily GDD values are accumulated (summed) throughout the season. Method differences calculating daily GDDs come into play from this point forward.

Method 1: Daily average temperatures are checked against the lower threshold, $T_{l}$. If the daily average temperature is colder than the lower threshold, $T_{l}$, the development will not go backward. Therefore, in order to eliminate negative accumulation, the average temperature is assumed to be the same as $T_{l}$ during days when the average temperature is less than the lower threshold temperature, $T_{l}$. Therefore, it would yield zero accumulation. The Applied Climate Information System (ACIS) is an example where this method is used for calculating GDD (ACIS, 2015 and Eggleston, 2015). The Applied Climate Information System (ACIS) was developed and is maintained by the National Oceanic and Atmospheric Administration (NOAA) Regional Climate Centers.

This method states that before Eq. 2 is applied, the following test should be executed

If $\frac{T_{x}+T_{n}}{2}<T_{l}$

Then $\frac{T_{x}+T_{n}}{2}=T_{l}$

Else $\frac{T_{x}+T_{n}}{2}=\frac{T_{x}+T_{n}}{2}$

This test assures that the daily average temperature is not less than the lower threshold temperature to eliminate negative accumulation. If it is less than the lower threshold, it will be assumed that it is set to the lower threshold.

During days when the maximum temperature is above the lower threshold and the minimum temperature is below the lower threshold temperature, this method underestimates the heat accumulation. The following example for corn is used in order to better demonstrate this underestimation. Since corn base temperature is $50^{\circ} \mathrm{F}$, then $T_{l}$ is 50 . If GDD needs to be calculated for a particular day when the daily maximum and minimum temperatures are $60^{\circ} \mathrm{F}$ and $40^{\circ} \mathrm{F}$ respectively, Eq. 2 can be used as follows:

$G D D=\frac{60+40}{2}-50=0$

It is obvious that the maximum temperature is $10^{\circ} \mathrm{F}$ greater than the base temperature for corn. Therefore, the daily temperatures must have stayed above the plant's development base temperature for some time throughout the day. But the method in Eq. 3 indicates zero heat accumulation for the plant development for this particular day. This would be a very typical case during the beginning and the end of a growing season. Thus, missing this portion of the accumulation during typical spring and fall days become important when accumulated throughout the season.

Certain corn hybrids are crafted to grow in certain regions determined by the average GDD accumulation for that region. These hybrids of corn are classified with a rating system called Relative Maturity (RM). RM is used to designate the relative time it takes for a certain hybrid to reach maturity (Ransom, 2004). Hybrids of short RM are adapted for colder climates, whereas hybrids of longer RM are 
better adapted for warmer climates. If method 1 is used to calculate GDD accumulations for a given season for longer and shorter RM hybrids, GDD accumulations that are underestimated will be a larger portion of the required GDD to mature for shorter RM hybrids than for longer ones. Therefore, Method 1 may underrepresent the corn plant's growth, especially during long spring seasons and in colder climates such as in North Dakota when the daily maximum and minimum temperatures fall on each side of the lower threshold temperature.

Method 2: In order to minimize the underestimation of heat accumulation during days when maximum and minimum temperatures fall on each side of the lower threshold temperature, both daily maximum and minimum temperatures are assumed to be at least the lower threshold temperature in two separate tests such as the following:

Test 1 If $T_{x}<T_{l}$

Then $T_{x}=T_{l}$

Else $T_{x}=T_{x}$

Test 1 assures that the daily maximum temperature is not less than the lower threshold temperature to eliminate negative accumulations. If it is less than the lower threshold, it will be assumed that it is set to the lower threshold.

Test 2 If $T_{n}<T_{l}$

Then $T_{n}=T_{l}$

Else $T_{n}=T_{n}$

Test 2 assures that the daily minimum temperature is not less than the lower threshold temperature to eliminate negative accumulations. If it is less than the lower threshold, it will be assumed that it is set to the lower threshold.

Test3 If $T_{x}>T_{u}$

Then $T_{x}=T_{u}$

Else $T_{x}=T_{x}$

Here, $T_{u}$ is the upper threshold at which the plant development is at a maximum and the plant does not further develop beyond this temperature. For corn, this value is $86^{\circ} \mathrm{F}$. Therefore another layer of tests was added to stop heat accumulations above $86^{\circ} \mathrm{F}$. Test 3 assures that the daily maximum temperature is not greater than the upper threshold temperature to eliminate exaggerated heat accumulations beyond the point where the plant development is at a maximum. If it is greater than the upper threshold, it will be assumed that it is set to the upper threshold.

With separate tests such as those above, the situation in the previous example used in Method 1 would have been different as follows:

$T_{x}=60$

$T_{n}=40$

$T_{l}=50$ (for corn)

Test $1 T_{x}>T_{l}$ then Then $T_{x}=60$ 
Test $2 T_{n}<T_{l}$

Then $T_{n}=50$

Test3 $T_{x}<T_{u}$

Then $T_{x}=T_{x}$

Substituting these new values into Eq. 2 yields the following:

$G D D=\frac{60+50}{2}-50=5$

Eq. 4

As seen in the example above, the second method captures the heat accumulation during the portion of the day when the actual temperature was above the base temperature for some time throughout the day. Even though it is not known how long the temperatures were above the threshold, Method 2 was able to capture a 5-degree-day accumulation which was neglected in Method 1. Based on this particular example, Method 1 underestimated the GDD by 5 Degree Days (DD) compared to Method 2. Since a portion of the day had conditions that are favorable to develop the plant during the period above the lower threshold temperature, Method 2 portrays a better picture of the actual conditions for corn on this day. The North Dakota Agricultural Weather Network (NDAWN) uses Method 2, also referred to as the "cut-off method" further explained in Neild and Newman (1990), and also used by Arguez et al., 2012.

In order to look at any systematic consistency, the authors compared seasonally accumulated GDDs in Fargo, ND, during the last 67-year period of growing seasons for corn from 1948 to 2014 using the daily maximum and minimum temperatures measured at Fargo Hector International Airport (the official Fargo climate location).

Figure 2 below is the result of the comparison between the two methods during the growing seasons from 1948 to 2014 in Fargo, ND. The authors restricted the accumulation period to 6-consecutive months from April through September based on the historical timing of planting and harvest practices in the Fargo area. Furthermore, the median first day of fall frost based on the 67 years of continuous historical weather data since 1948 in Fargo is September 25. Therefore the authors did not want to accumulate heat after plant development ceases. The vertical axis in Figure 2 is the seasonally Accumulated Growing Degree Days (AGDD) from April 1 through September 30. Blue and orange lines represent methods 1 and 2 respectively. The AGDDs are calculated seasonally by summing the daily GDDs calculated using Eq. 5 below.

$A G D D=\sum_{i=A p r 1}^{\operatorname{Sep} 30} G D D_{i}$

Eq. 5

In Eq. 5, AGDD is the Accumulated Growing Degree Days for corn (50-degree base temperature), and GDD $i$ is the individual Growing Degree Days in $i^{\text {th }}$ day where $i$ ranges between April 1 and September 30. Daily growing degree days $\left(G D D_{i}\right)$ are calculated using Eq. 3 for Method 1 and Eq.4 for Method 2.

\section{Conclusions}

1. Figure 1 shows that the seasonally accumulated corn GDDs calculated by Method 1 is systematically lower than that calculated by Method 2.

2. The average difference in magnitude between the two methods is 173 degree days (DD) per season with the maximum of 287 DD in 1993, and with the minimum of 41.5 DD in 2013. 
3. The average difference in magnitude between the two methods of 173 degree days (DD) per season is equivalent to 21 days for a corn hybrid maturing on September 15 . The difference is equivalent to a longer period for a later maturity dates using the average GDD accumulation in this period based on Fargo's historic daily GDD data (Average daily GDD on September 15 is 10.5 Degree Days and it lessens by 0.25 DD per day until the end of growing season when the daily GDD becomes negligible). Method 1 could no longer be applicable for a corn hybrid to mature later than October 10, because the rest of the season would not be warm enough to make up even the minimum difference of 41.5 DD based on Fargo's normal daily GDD data after October 10.

4. The maximum difference of 287 DD in 1993 is approximately $13 \%$ of required GDD for a typical corn hybrid planted in Fargo, ND. (A typical corn hybrid for the Fargo area is $90 \mathrm{RM}$, which requires at least 2151 GDD accumulation per season (Ransom, 2004); therefore, $287 / 2151=0.133$ or $13 \%$ )

5. The magnitude of the difference is directly proportional to the length of spring and fall seasons when the daily maximum temperature is above, and the daily minimum temperature is below the lower threshold (base) temperature $\left(T_{l}\right)$ (number of shoulder days). The graphic below in Figure 3 indicates the correlation $\left(r^{2}=0.65\right)$ between the magnitude of the difference between the methods and number of shoulder days in a given season when the daily maximum temperature is above, and the daily minimum temperature is below the lower threshold temperature $\left(T_{l}\right)$.

6. The most number of shoulder days was 87 days in 1973 (1993 was the year with the second largest number of shoulder days), and the least number of shoulder days was 41 in 2013 which also corresponds to the season with the least difference between the two methods in GDD accumulation calculation.

7. The authors recommend using Method 2 over Method 1 to calculate Growing Degree Days since Method 2 tends to capture the heat accumulation in the beginning and the end of the growing seasons far better than the Method 1. Furthermore, the proportion of the difference to the required GDD proportion accumulation for a corn hybrid that is typical to a region is greater in colder climates than warmer climates since warmer corn hybrids require greater GDD accumulations (Neild et al., 1990).

8. The authors caution the readers that these methods are an estimation of heat units that are correlated with plant development stages and strongly encourages the agronomists and farmers to check with actual conditions in the field before making decisions.

9. Finally, the authors recommend using hourly rather than daily temperature data to estimate daily GDDs which better accounts for the plant development rate in response to heat accumulation. As automated weather observations have advanced and become an integrated part of the nation's climate inventory, using this method should not be as difficult as it was in the past.

\section{References:}

ACIS, 2015. Applied Climate Information System. http://www.rcc-acis.org/ . Page last accessed on October 19, 2015.

Arguez, A., I. Durre, S. Applequist, R. S. Vose, M. F. Squires, X. Yin, R. R. Heim, Jr., and T. W. Owen, 2012: NOAA's 1981. 2010 U.S. Climate Normals: An Overview. Bulletin of the American Meteorological Society. November 2012. DOI:10.1175/BAMS-D-11-00197.1. 1687-1697

Badh, A., and F. A. Akyuz, 2010. Evaluating Trend Changes in Annual Accumulated Growing Degree Days for Corn in the Northern Plains, United States of America. International Journal of Climate Change: Impacts and Responses 2010 Vol. 2 No. 2 pp. 127-136. 
Eggleston, K., 2015: Electronic Communication. Eggleston, K. to A. Akyuz. Re: GDD Calculations. October 19, 2015.

Gilmore, E.C., and J. S. Rogers. 1958. Heat Units as a Method of Measuring Maturity in Corn. Agronomy Journal. Vol. 50 No. 10, p. 611-615.

Miller, P, W. Lanier, and S. Brandt, 2001. Using Growing Degree Day to Predict Plant Stages. MT State University Extension Service Montguide. MT200103 AG 7/2001.

(http://www.ipm.montana.edu/Training/PMT../2006/mt200103.pdf)

NDAWN, 2015. North Dakota Agricultural Weather Network. North Dakota State University. URL: http://ndawn.ndsu.nodak.edu/. Page last accessed on August 10, 2015.

Neild, R. E., and J. E. Newman, 1990. Growing Season Characteristics and Requirements in the Corn Belt. $\mathrm{NCH}-40$. Cooperative Extension Service, Purdue University, W. Lafayette, IN.

Ransom, J. 2004. Basics of Corn Production in North Dakota. NDSU Extension Services. North Dakota State University. July 2004. A-834. 


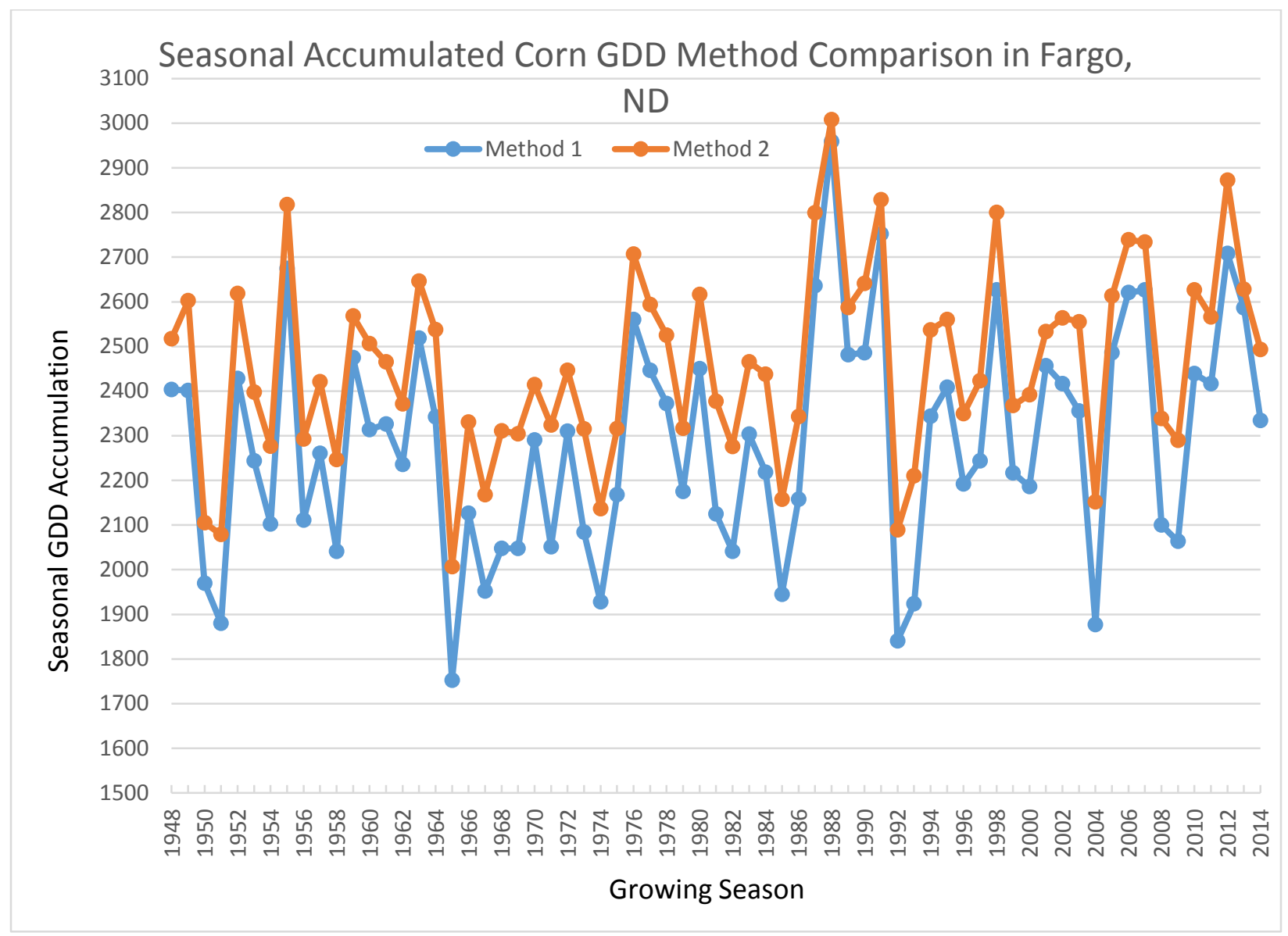

Figure 2. Seasonal Accumulated Corn Growing Degree Days during the last 67 years from 1948 to 2014 in Fargo, ND. Method 1 (Blue Bars) and Method 2 (Orange Bars). (Average difference is 173 Degree Days per Season) 


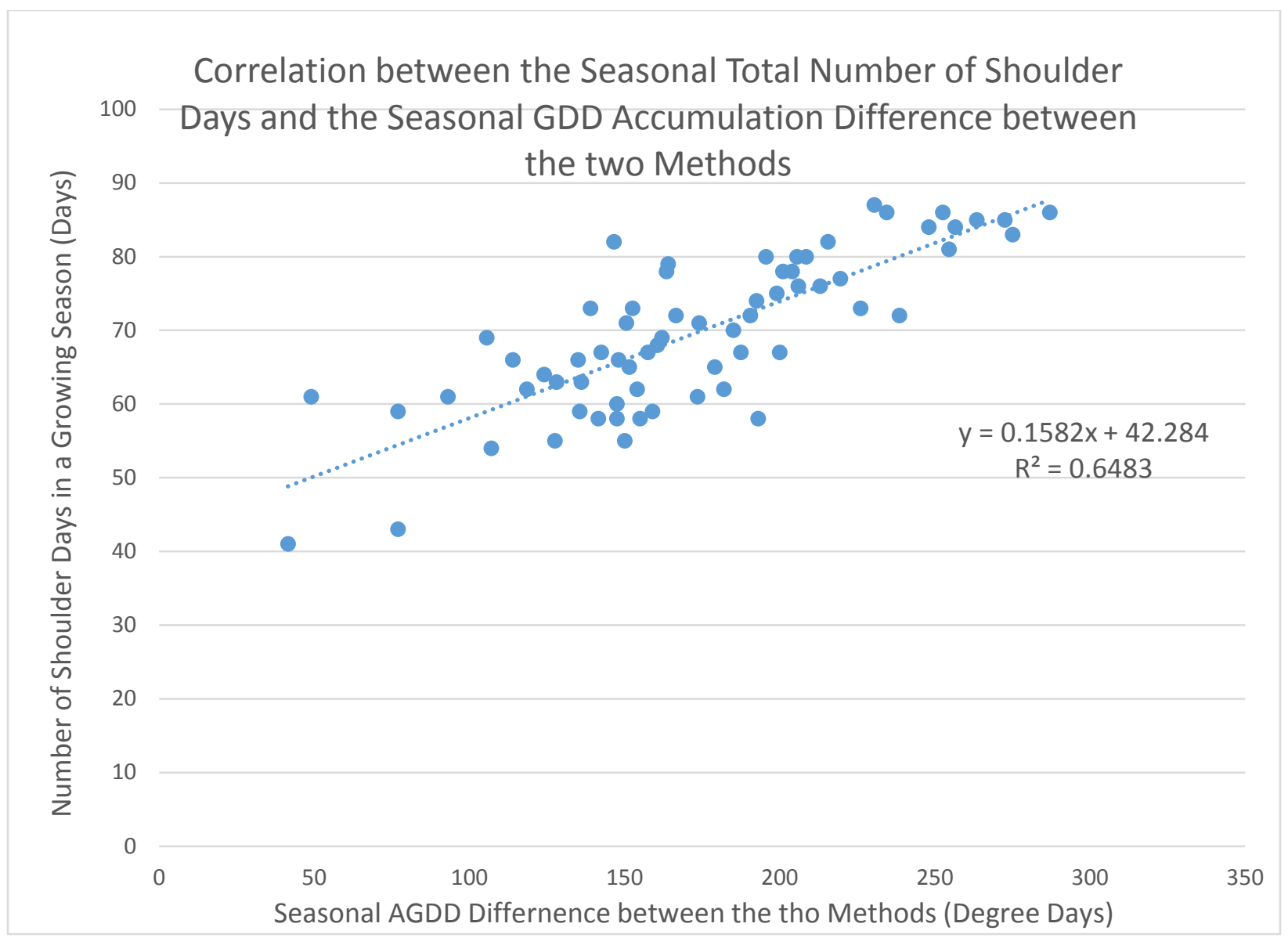

Figure 3. Correlation between Seasonal Difference in Accumulated Growing Degree Days between the two Methods, and the Number of Shoulder Days (Number of Days when the Daily Maximum Temperature is at least $50^{\circ} \mathrm{F}$ and the Daily Minimum Temperature is Less than $50^{\circ} \mathrm{F}$.) 